\title{
COVID-19 in the act: incidental 18F-FDG PET/CT findings in asymptomatic patients and those with symptoms not primarily correlated with COVID-19 during the United Kingdom coronavirus lockdown
}

\author{
Richard Halsey ${ }^{1}$ (D) Dimitrios Priftakis ${ }^{1} \cdot$ Strachan Mackenzie ${ }^{2} \cdot$ Simon Wan $^{1} \cdot$ Laura M. Davis $^{1} \cdot$ David Lilburn $^{1}$. \\ Andrew Thornton ${ }^{1} \cdot$ Nikolaos Papathanasiou $^{3} \cdot$ Gopinath Gnanasegaran ${ }^{4} \cdot$ Jamshed Bomanji $^{1}$
}

Received: 3 June 2020 / Accepted: 23 July 2020 / Published online: 5 August 2020

(C) Springer-Verlag GmbH Germany, part of Springer Nature 2020

\begin{abstract}
Purpose The emergence of the novel SARS-CoV-2 pathogen and lethal COVID-19 disease pandemic poses major diagnostic challenges. The study aims to describe the spectrum and prevalence of thoracic and extrathoracic incidental findings in patients who have undergone 18F-FDG PET/CT during the first 3 weeks of the COVID-19 UK lockdown.

Methods This is a single-centre retrospective controlled observational study. 18F-FDG PET/CT scans $(n=160)$ acquired from 23/3/2020 to 9/4/2020 were retrospectively reviewed for incidental findings in the lungs and extrapulmonary sites (heart, nasal sinuses, parotid and salivary glands, colon, large vessels, renal cortex, brain, spleen and testes). A date-matched control group $(n=205)$ of patients from 2019 was used for comparison.

Results The total prevalence of suspicious findings was 26/160 (16.25\%). Fifteen patients presented with incidental findings in the lungs, while eleven patients had only non-pulmonary incidental findings. There was a significant increase in the appearance of incidental 18F-FDG PET/CT findings during the 2nd week $(\mathrm{OR}=3.8)$ and $3 \mathrm{rd}$ week $(\mathrm{OR}=7.6)$ in relation to the 1st week. There was a significant increase in the average maximum standardised uptake values (SUVmax) in the parotid/salivary glands of patients scanned during week 2 in relation to week $1(p=0.036)$. There was no significant difference in the prevalence of
\end{abstract}

Richard Halsey, Dimitrios Priftakis, Strachan Mackenzie, Simon Wan,

Laura M. Davis, David Lilburn, Andrew Thornton, Nikolaos

Papathanasiou, Gopinath Gnanasegaran and Jamshed Bomanji

contributed equally to this work.

This article is part of the Topical Collection on Infection and inflammation

Electronic supplementary material The online version of this article (https://doi.org/10.1007/s00259-020-04972-y) contains supplementary material, which is available to authorized users.

Richard Halsey

rhalsey@nhs.net

Dimitrios Priftakis

dimitris.priftakis@nhs.net

Strachan Mackenzie

strachan.mackenzie@nhs.net

Simon Wan

mwan@nhs.net

Laura M. Davis

lauramaydavis@nhs.net
David Lilburn

david.lilburn@nhs.net

Andrew Thornton

andrew.thornton11@nhs.net

Nikolaos Papathanasiou

nikopapath@googlemail.com

Gopinath Gnanasegaran

gopinath.gnanasegaran@nhs.net

Jamshed Bomanji

jamshed.bomanji@nhs.net

Extended author information available on the last page of the article 
incidental findings compared to the control group, but the number of pulmonary vs. extrathoracic findings was different between the two populations.

Conclusion The study provides a novel base of evidence to identify asymptomatic patients and those without symptoms strongly associated with COVID-19 with incidental 18F-FDG PET/CT findings suspicious of SARS-CoV-2 infection during the initial stages of the pandemic.

Keywords COVID-19 · SARS-CoV-2 $\cdot$ Imaging $\cdot$ 18F-FDG PET/CT $\cdot$ Incidental findings $\cdot$ Asymptomatic $\cdot$ Extrathoracic

$\begin{array}{ll}\text { Abbreviations } & \\ \text { ALCL } & \text { Anaplastic large cell lymphoma } \\ \text { BSTI } & \text { British Society of Thoracic Imaging } \\ \text { CLL } & \text { Chronic lymphocytic leukaemia } \\ \text { CTPA } & \begin{array}{l}\text { Computed tomography } \\ \text { pulmonary angiogram }\end{array} \\ & \text { Diffuse large B cell lymphoma } \\ \text { DLBCL } & \text { European Association of Nuclear Medicine } \\ \text { EANM } & \text { Fluorodeoxyglucose } \\ \text { FDG } & \text { Gastrointestinal stromal tumour } \\ \text { GIST } & \text { Hodgkin lymphoma } \\ \text { HL } & \text { Interquartile range } \\ \text { IQR } & \text { Non-Hodgkin lymphoma } \\ \text { NHL } & \text { Picture archiving and communication } \\ \text { PACS } & \text { system } \\ & \text { Polymerase chain reaction } \\ \text { PCR } & \text { Positron emission tomography/ } \\ \text { PET/CT } & \text { computed tomography } \\ & \text { Primary mediastinal B cell lymphoma } \\ \text { PMBCL } & \text { Pyrexia of unknown origin } \\ \text { PUO } & \text { Severe acute respiratory syndrome } \\ \text { SARS } & \text { Severe combined immunodeficiency } \\ \text { SCID } & \text { Severe acute respiratory syndrome } \\ \text { SARS-CoV-2 } & \text { coronavirus 2 } \\ & \text { Standards for Reporting Studies of } \\ \text { STARD } & \text { Diagnostic Accuracy } \\ & \text { Standardized uptake value, SUVmax } \\ \text { SUV } & \text { being the maximum value } \\ & \end{array}$

\section{Introduction}

The emergence of the novel pathogen SARS coronavirus 2 (SARS-CoV-2) and the lethal human coronavirus disease 2019 (COVID-19) it causes to pandemic proportions is such that it poses major challenges for the diagnostic process.

As no vaccine against SARS-CoV-2 or specific treatment for COVID-19 has yet been shown to be effective, many countries have introduced 'lockdown' measures to mitigate community transmission of the virus. The UK adopted such measures on the 23rd of March 2020 [1].

It is recognised that a number of patients with SARS-CoV2 infection can have a subclinical phase to their disease course, or even remaining asymptomatic throughout. The exact incidence of this remains a matter of debate. These patients present particular challenge in theoretical epidemiological modelling of disease transmission (which in turn informs policy decisions), as well as in practical aspects of the running of healthcare facilitates to protect non-infected patients and staff [2].

Special attention has been proposed to the identification of incidental findings in 18F-FDG PET/CT studies which could raise the suspicion of COVID-19 in asymptomatic but potentially infective patients, and to the subsequent management of these patients with regards to the need for equipment cleaning and patient isolation [3].

The purpose of this study is to describe the demographic, clinical and 18F-FDG PET/CT features of patients with cancer and other acute indications for 18-F-FDG PET/CT imaging who were undergoing a baseline study for staging or were having an interval scan during therapy in a large teaching hospital in London designated as a COVID-19 hub during the initial 3 weeks of UK lockdown, and to compare them with a date-matched control group of pre-SARS-CoV-2 emergence (Spring 2019) patients. In addition, potential extrathoracic manifestations of the infection and its complications [4] were sought.

\section{Materials and methods}

\section{Study design}

This is a single-centre retrospective controlled observational study.

\section{Patient selection criteria}

As a response to the increasing admission needs, University College London Hospital (UCLH) has been designated a COVID-19 hub. The Institute of Nuclear Medicine adapted to the situation by drawing up plans to maximise patient and staff safety without compromising the services to cancer and other acute patients, in line with government guidelines [3]. Universal testing for COVID-19 was not a precondition for out-patient appointments.

The Institute of Nuclear Medicine at UCLH has a workload of just over 18,000 scans annually, of which approximately 
7500 are clinical PET imaging scans. In preparation for the COVID-19 pandemic, all non-urgent and research scans were postponed $[3,5]$ for 12 weeks. The PET/CT service was running for the urgent staging of new cancer patients, for treatment response assessment, particularly in lymphoma patients, for urgent restaging of patients with high suspicion for recurrence and for selected urgent cases of infection/inflammation assessment (e.g. suspected vasculitis or fever of unknown origin).

11 patients were screened on the day before the scan for symptoms (such as new cough or fever), for contact with confirmed or suspected cases in the last 14 days and for recent travel abroad either by telephone (outpatients) or by contact with the referring clinician (inpatient), according to UCLH Trust guidance. Patient responses were recorded in Epic, the electronic health records systems used across the UCLH Trust. On arrival in the department, patients were again assessed for symptoms and temperature before proceeding to the preparation area and radiotracer injection. Only patients with no symptoms or with mild symptoms not primarily correlated with COVID-19 and not included in the screening Government [6] and Trust guidance at the time of the study, and who were not in isolation at home or in the hospital were allowed to proceed with the procedure. Rare exceptions have been made depending on the clinical urgency of the scan after consultant-to-consultant referral and discussion, and with the appropriate provisions for patient transportation, staff safety measures and deep cleaning of the scanning room after the procedure. Inclusion and exclusion criteria are described in Table 1.

Table 1 Inclusion and exclusion criteria of patients who were undergoing a baseline study for staging or were having an interval PET/ CT scan during therapy in a large teaching hospital in London designated as a COVID-19 hub during the initial three weeks of UK lockdown

Inclusion criteria
Patients referred for $18 \mathrm{~F}-\mathrm{FDG}$ PET/CT to UCLH
Time period of study: $23 / 3 / 20-9 / 4 / 20$
Patients with no reported symptoms or temperature at pre-procedure
screening call
Patients with normal temperature on arrival
Exclusion criteria
Patients reporting cough, fever, severe gastrointestinal symptoms at
pre-procedure screening call
Outpatients and inpatients in isolation according to NHS guidance for
COVID-19
Patients with temperature $>37.8{ }^{\circ} \mathrm{C}$ on arrival
Patients with positive SARS-CoV-2-PCR test
Blood glucose $>11$ mmol/L or $>200$ mg/dL
Patients $<18$ years old
Limited field of view scans such as brain-only scans for epilepsy
External 8 F-FDG PET/CT scans (performed elsewhere) received by
IEP in PACS

\section{F-FDG PET/CT image acquisition}

Scanning was performed according to EANM guidelines [7]. The scans were performed in a GE Discovery 710 PET/CT scanner and in a Siemens Biograph Vision 600 PET/CT scanner. Cross-calibration of the scanners had been performed in order to acquire comparable maximum standardised uptake values (SUVmax).

\section{Data collection}

The patients' images were retrieved from the UCLH PACS, and the patients' demographic and clinical information were retrieved from the electronic health record system (Epic) used in UCLH. Only the authors who have clinical access to PACS and Epic as per their contract with UCLH took part in data collection and processing (RH, DP, LMD, DL, AT, SW, JB), compliant with Trust confidentiality in the health and social care information guidance policy. The scan selection process is shown in the STARD flow chart in Fig. 1.

The data was anonymised and a secured standardised spreadsheet was used for entering age, sex, ethnicity (where stated), indication, COVID-19 shielding status, clinical signs and symptoms, physical findings, laboratory findings, 18FFDG PET/CT findings and follow-up data for each patient. The COVID-19 shielding status follows the NHS digital guidance for shielding patients at high risk against severe complications [8].

\section{SARS-CoV-2 testing}

Universal testing for SARS-CoV-2 was not at the time performed for all inpatients and not a precondition for out-patient appointments. Decision for testing was made by the clinical teams based on prevailing NHS guidance at the time. When this was done, samples were collected using a combined throat and nose swab and a direct or real-time (Hologic Panther Fusion Assay) PCR assay was performed. Results were captured via the Trust Epic pathology system. Twelve patients in the study cohort had PCR testing within a week of the $18 \mathrm{~F}$ FDG PET/CT.

\section{Interpretation of 18F-FDG PET/CT scans}

The 18F-FDG PET/CT scans were retrospectively reviewed blind to the original clinical report. The researchers aimed to identify the incidental appearance of lung changes, which were classified based on the CT pattern suggestions in the British Society of Thoracic Imaging (BSTI) guidelines (Table 2) [9]. As BSTI interpretation partly depends on the presence and severity of clinical finding, modifications to account for the lack of clinical context in the majority of the current study's patients were asymptomatic or without major 

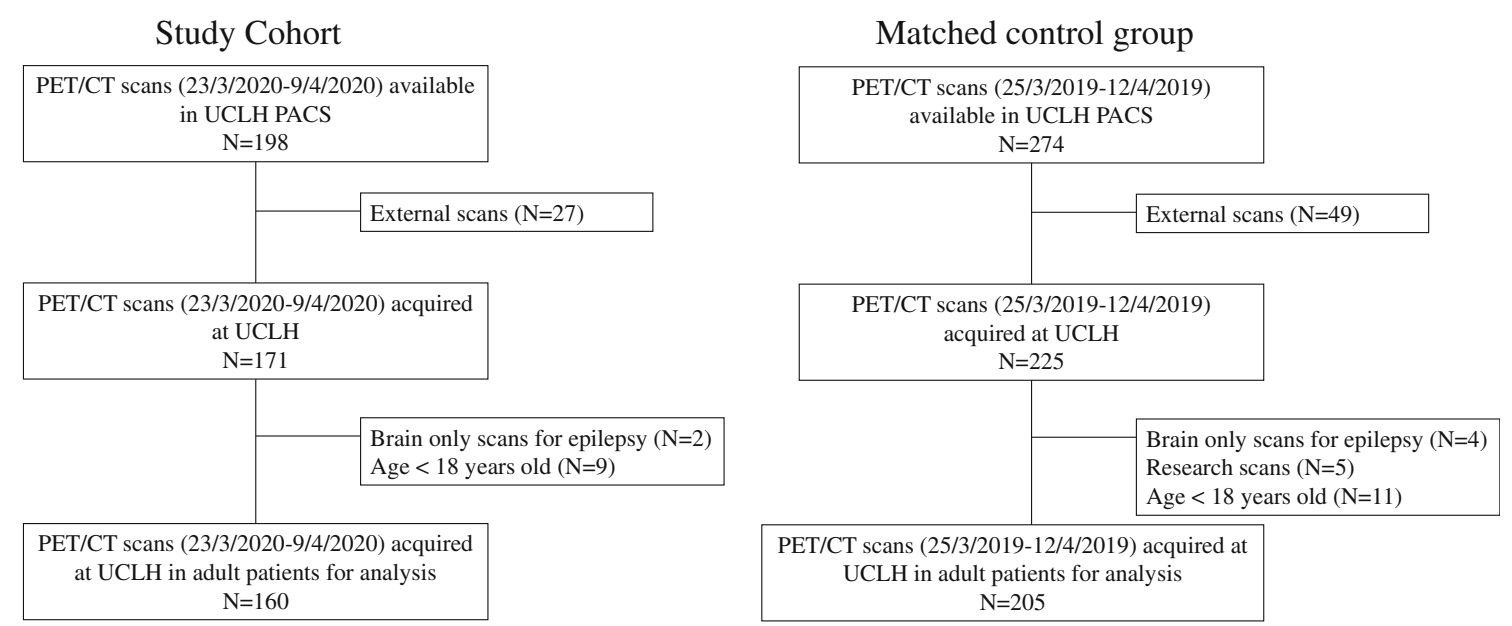

Fig. 1 STARD flow chart of 18F-FDG PET/CT scan selection for study cohort (left) and matched control group (right). [UCLH PACS = University College London Hospital Picture Archiving and Communication System]

symptoms as defined by Public Heath UK guidance were adopted $[6,9]$. All the incidental findings fitting in the first two BSTI defined categories (Classic, Probable/Intermediate) were registered as suspicious COVID-19 cases and the SUVmax of the lesions was measured (Supplementary material, Table S2). Potential suspicious alterations of FDG uptake and SUVmax values were documented in extrathoracic organs in line with previously published non-respiratory involved sites in these patients (Supplementary material, Table S1) [4, 10-13] .

For the interpretation of the findings in the aforementioned extrathoracic sites, a combination of increased 18F-FDG uptake and abnormal radiological features was followed. Incidental findings (such as focal or diffuse colonic uptake in patients not on metformin or increased salivary or tonsillar uptake in patients without pathological or previous involvement of these sites) were registered as suspicious after blinded doublereading of the study by the investigators.

A control cohort was defined, including adult patients who had 18F-FDG PET/CT scans during the same time interval in 2019, applying the same inclusion and exclusion criteria where applicable (Fig. 1). The control group serves as a reference for the prevalence and type of incidental findings. Date matching was selected in order to account for seasonal variation of respiratory infections. It is assumed that the case group and the control group have comparable demographic and clinical characteristics. No statistical comparison of the data distribution for age, sex, ethnicity and clinical indication was performed as there is no intended comparison between the case and control cohort dependent on these variables and no inclusion or exclusion criterion on the basis of patient profile. Similar interpretation criteria were followed for the control 18F-FDG PET/CT scans.
Table 2 The British Society of Thoracic Imaging, classification of incidental lung patterns observed on CT. The CT component of 18F-FDG PET/CT was used for this classification in this study (source: The British Society of Thoracic Imaging website, see ref. [8]

\begin{tabular}{ll}
\hline $\begin{array}{l}\text { BSTI-Defined CT chest } \\
\text { pattern }\end{array}$ & Description \\
\hline Classic COVID-19 & $\begin{array}{l}>1 \text { lower lobe predominant, peripheral predominant, unilateral or bilateral foci of } \\
\text { ground-glass opacities } \\
+/- \text { one or more of the following: } \\
\text { Crazy paving } \\
\text { Peripheral consolidation }\end{array}$ \\
& $\begin{array}{l}\text { Air bronchogram } \\
\text { Reverse halo/perilobular pattern } \\
\text { Lower lobe predominant mix of bronchiocentric and peripheral consolidation or } \\
\text { reverse halo/perilobular pattern with scarce ground-glass opacities. }\end{array}$ \\
& $\begin{array}{l}\text { Findings compatible with known disease, pre-existing changes in previously } \\
\text { available imaging before the pandemic or patterns not fitting in the first two } \\
\text { categories. }\end{array}$ \\
& $\begin{array}{l}\text { Normal } \\
\text { Normal }\end{array}$ \\
\hline
\end{tabular}


Table 3 Patient demographic characteristics within study and control group

\begin{tabular}{lll}
\hline Characteristics & Study group $(n=160)$ & Control group $(n=205)$ \\
\hline Age & & \\
$\quad$ MEdian (IQR) (yrs) & $62.2(49.7-62.8)$ & $62(50.5-72)$ \\
Sex & & \\
Female & 80 & 99 \\
Male & 80 & 106 \\
Self-reported ethnicity & \\
BAME & 21 & 26 \\
White & 65 & 84 \\
Not stated & 74 & 95 \\
\hline
\end{tabular}

\section{Data analysis/statistics}

The relationship between the appearance of suspicious findings and independent variables, such as gender, age and date of scan (i.e. 1st, 2 nd or 3 rd week within the defined date range), was explored with a logistic regression model. The SUVmax values from each of the aforementioned extrathoracic sites were also correlated with the week of the scan to look for potential average increase with time. A simple linear regression model was applied with SUVmax from each specific site as the dependent variable and the week of the scan as independent categorical variable.

Comparison of the prevalence of incidental findings between the 2020 cohort and the 2019 control patient cohort was performed with a chi-square test. A qualitative comparison of the patterns of incidental findings between the two cohorts was also made.

\section{Patient follow-up}

Follow-up information for patients over the subsequent 2 weeks was retrieved from Epic for follow-up encounters with the clinical/referring team (either in person or remotely).
Table 4 Clinical indications for 18 F-FDG PET/CT scans in study group and control patients

\begin{tabular}{|c|c|c|}
\hline indication/comorbidity & Study group $(n=160)$ & Control group $(n=205)$ \\
\hline Haematological malignancy & $n=55$ & $n=81$ \\
\hline \multicolumn{3}{|l|}{ (Subtype) } \\
\hline HL & 12 & 9 \\
\hline NHL & 23 & 33 \\
\hline Lymphoma, other & 7 & 17 \\
\hline CLL & 1 & 3 \\
\hline Myeloma & 10 & 14 \\
\hline Plasmacytoma & 1 & 0 \\
\hline GIST & 1 & 2 \\
\hline Other & 0 & 3 \\
\hline Oncology: & $n=94$ & $n=99$ \\
\hline Lung tumour & 33 & 30 \\
\hline Pulmonary nodule(s) & 3 & 17 \\
\hline Gastrointestinal & 23 & 14 \\
\hline Genitourinary & 13 & 12 \\
\hline Breast & 8 & 5 \\
\hline Other primary & 14 & 21 \\
\hline Other & $n=11$ & $n=25$ \\
\hline PUO & 2 & 2 \\
\hline Connective tissue disorder & 1 & 1 \\
\hline Query infective focus & 2 & 10 \\
\hline Vasculitis & 2 & 3 \\
\hline Sarcoid & 1 & 1 \\
\hline Other & 3 & 8 \\
\hline
\end{tabular}

IQR interquartile range, BAME black, Asian and minority ethnic, $n$ number of patients

$H L$ Hodgkins lymphoma, NHL non-Hodgkins lymphoma, $C L L$ chronic lymphocytic leukaemia, GIST gastrointestinal stromal tumour, $P U O$ pyrexia of unknown origin. 'Other primary' encompasses (number, in study group, number, in control group): sarcoma $(3,7)$, neuroendocrine neoplasm $(1,1)$, skin $(1,0)$, thyroid $(0,2)$, thymic $(0,2)$, brain $(0,1)$ and cancer of unknown primary $(6,8)$ 
Table 5 Symptoms on clerking as documented on pre-scan assessment in the nuclear medicine department on the day. Patients did not report significant potential COVID-19-related symptoms on screening days prior to the scan either by phone (outpatients) or via clinical team (inpatients). Dyspnoea was reported only by those with preexisting pulmonary pathology such as lung cancer. For the single inpatient with fever (Fig. 7), 18FFDG PET/CT was performed as part of screening for pyrexia of unknown origin, initial viral PCR testing (including SARS-CoV-2) was negative prior to scanning

\begin{tabular}{lc}
\hline Symptoms on clerking & Number of cases $(n=160)$ \\
\hline Fever & 1 \\
Dyspnoea & 9 \\
Cough & 2 \\
Coryzal/hayfever & 5 \\
Fatigue & 2 \\
Chest pain & 2 \\
History of chest symptoms (no symptoms on clerking) & 3 \\
Anorexia/weight loss & 2 \\
GI upset & 0 \\
Abdo pain & 3 \\
Headache & 1 \\
Back pain & 3 \\
Thrombus & 1 \\
Other & 7 \\
None & 116 \\
Not recorded & 3 \\
\hline
\end{tabular}

There was one recorded death (cause noted as pre-existing neoplastic disease-related, no mention in the notes of pneumonia), and no recorded admissions of outpatients to UCLH for suspected COVID-19 pneumonia.

\section{Results}

Patient demographics clinical indication, symptoms reported on the day of the scan and COVID-19 shielding status are presented in Table 3, 4, 5 and 6.

The vast majority $(116 / 160,73 \%)$ of patients were asymptomatic on the day of the scan, and the remaining reported only mild symptoms not strongly associated with COVID19 and not included in the screening Government [6] and Trust guidance at the time of the study (40/160, 25\%). One in-patient had 18F-FDG PET/CT as part of screening for pyrexia of unknown origin, and had a negative viral screening (including SARS-CoV-2 PCR) prior to the scan. In a small number, symptoms were not recorded (3/160, 2\%).
The total prevalence of suspicious findings was 26/160 $(16.25 \%)$. Of those 26 patients, 15 presented incidental findings in the lungs while 11 patients had only non-pulmonary incidental findings. A detailed description of the patients and the incidental findings on 18F-FDG PET/CT are given in supplementary material Table S2 and Table S3.

The prevalence of incidental findings was $3 / 59(5 \%)$ in the 1st week, 9/53 (17\%) in the 2nd week and 14/48 (29.1\%) in the 3 rd week. Logistic regression analysis showed a significant increase in the appearance of incidental 18F-FDG PET/ CT findings during the 2 nd and $3 \mathrm{rd}$ week in relation to the $1 \mathrm{st}$ week $(\mathrm{OR}=3.8, p=0.05$ and $\mathrm{OR}=7.6, p=0.002$ for the 2 nd and 3 rd week, respectively) (Table 7 ).

Figure 2 shows a cumulative graph of the appearance of incidental 18F-FDG PET/CT findings in our patient group superimposed onto a cumulative graph of the confirmed COVID-19 cases in the UK in the same time interval [14].

Logistic regression analysis did not show any significant association of the appearance of suspicious imaging findings on 18F-FDG PET/CT with gender, for males compared to

Table 6 Symptomatic and COVID-19 shielded status and clinical COVID-19-PCR testing result of study group by comorbidity

\begin{tabular}{|c|c|c|c|c|c|c|c|}
\hline \multicolumn{8}{|l|}{ Study group } \\
\hline Comorbidity & $n=$ & Symptomatic & $\begin{array}{l}\text { COVID } \\
\text { shielded }\end{array}$ & Tested & $\begin{array}{l}\text { Positive } \\
\text { test }\end{array}$ & $\begin{array}{l}\text { Symptomatic with } \\
\text { incidental findings on PET/ } \\
\text { CT }\end{array}$ & $\begin{array}{l}\text { Asymptomatic with } \\
\text { incidental findings on PET/ } \\
\text { CT }\end{array}$ \\
\hline $\begin{array}{l}\text { Haematological } \\
\text { malignancy }\end{array}$ & 91 & 14 & 33 & 4 & 0 & 2 & 2 \\
\hline Oncology malignancy & 55 & 3 & 34 & 4 & 1 & 2 & 3 \\
\hline Other & 14 & 9 & 9 & 4 & 0 & 2 & 2 \\
\hline
\end{tabular}


Table 7 Mean maximum standardised uptake values (SUV max) and standard deviation of measured regions of interest in the whole study patient population $(N=160)$. Mean maximum standardised uptake values (SUVmax) and standard deviations of measured regions of interest per week of scanning. $P$ values of the pairwise comparisons between weeks - results from the regression models

\begin{tabular}{|c|c|c|c|c|c|c|c|c|}
\hline & \multicolumn{3}{|l|}{ Whole patient population } & \multicolumn{3}{|c|}{ SUVmax values (mean \pm SD) per week } & \multicolumn{2}{|c|}{ Pairwise $p$ values } \\
\hline & SUVmax values (mean \pm SD) & Median & IQR & 1 st week & 2nd week & 3rd week & week 2 vs. 1 & week 3 vs. 1 \\
\hline Tonsillar uptake & $4.85 \pm 2.01$ & 4.35 & $3.4-5.8$ & $4.8 \pm 1.9$ & $5.0 \pm 1.8$ & $4.7 \pm 2.3$ & 0.733 & 0.753 \\
\hline Salivary gland uptake & $2.66 \pm 1.22$ & 2.4 & $1.9-2.9$ & $2.4 \pm 1.3$ & $2.9 \pm 1.2$ & $2.6 \pm 1.1$ & 0.036 & 0.416 \\
\hline Mediastinal uptake & $2.39 \pm 0.51$ & 2.35 & $2.05-2.7$ & $2.4 \pm 0.5$ & $2.4 \pm 0.4$ & $2.5 \pm 0.6$ & 0.893 & 0.325 \\
\hline Gastric uptake & 3. $58 \pm 1.12$ & 3.3 & $2.8-4.3$ & $3.4 \pm 1.3$ & $3.7 \pm 1.0$ & $3.6 \pm 1.0$ & 0.217 & 0.399 \\
\hline Liver uptake & $3.20 \pm 0.67$ & 3.1 & $2.8-3.55$ & $3.2 \pm 0.7$ & $3.1 \pm 0.6$ & $3.3 \pm 0.7$ & 0.520 & 0.236 \\
\hline Renal uptake & $4.03 \pm 0.94$ & 4.1 & $3.3-4.7$ & $4.1 \pm 0.9$ & $4.1 \pm 1.0$ & $3.9 \pm 0.9$ & 0.727 & 0.490 \\
\hline Colonic uptake & $5.05 \pm 2.68$ & 4.1 & $3.1-6.4$ & $4.7 \pm 2.4$ & $5.1 \pm 3.0$ & $5.7 \pm 2.8$ & 0.502 & 0.135 \\
\hline
\end{tabular}

females $(\mathrm{OR}=1.4, p=0.397)$ or age $(\mathrm{OR}=0.99, p=0.875)$ or ethnicity $(\mathrm{OR}=2.7, p=0.095)$.

Descriptive statistics for the SUVmax measurements are shown in Table 7. Regression analysis showed a significant increase of SUVmax in the parotid/salivary glands during week 2 in relation to week $1(p=0.036)$. However, this observation was not repeated in week 3 , where there was no significant difference in parotid uptake in comparison to week 1 ( $p=0.416)$ (Table 7 and Fig. 3). No other significant increase of average SUVmax with time was observed in any of the remaining measured anatomical sites (supplementary material, Figures S1-S6).

The total prevalence of incidental findings in the control group from 2019 was 33/205 (16.1\%). The chi-square test of independence to examine the relationship between the frequencies of incidental findings in the 2019 and 2020 patient groups did not show a significant relationship $\left(X^{2}=0.001, p=0.968\right)$. Thoracic findings in the control group were further categorised in terms of imaging review, clinical history and follow-up imaging. Five were areas of new disease in the context of pre-existing lung cancer, 1 was detection of a new pulmonary metastasis from a breast cancer primary, 2 were areas of aspiration that resolved on subsequent imaging, 2 were areas of post-surgical change that became non-avid on follow-up and 1 was inflammation in an apical distribution that would be classed as not typical by BSTI guidelines. Of the remaining $1018 \mathrm{~F}-\mathrm{FDG}$ PET CT findings, 4 would be categorised under BSTI criteria as classic and 6 as probable/indeterminate.

However, a higher number of incidental pulmonary findings vs. extrathoracic findings were observed during the study time period which was reversed in the 2019 control group where a higher number of extrathoracic findings were observed (Fig. 4). Finally, no specific trend for increase of incidental findings with time was observed in the 2019 control group.
Fig. 2 Cumulative graph of incidental 18F-FDG PET/CT findings in our cohort (red line) plotted against the cumulative graph of the confirmed COVID19 cases in the UK at the same time interval (blue line). The $Y$ axis represents the cumulative number of cases

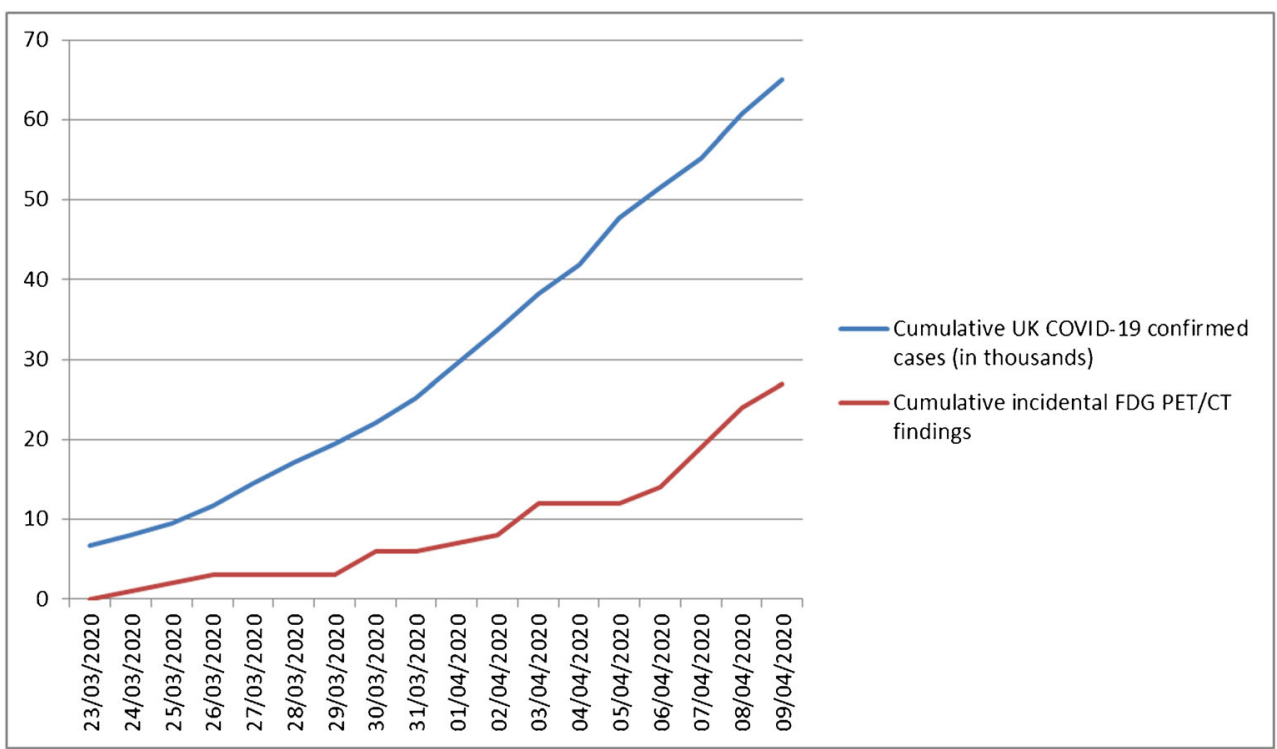




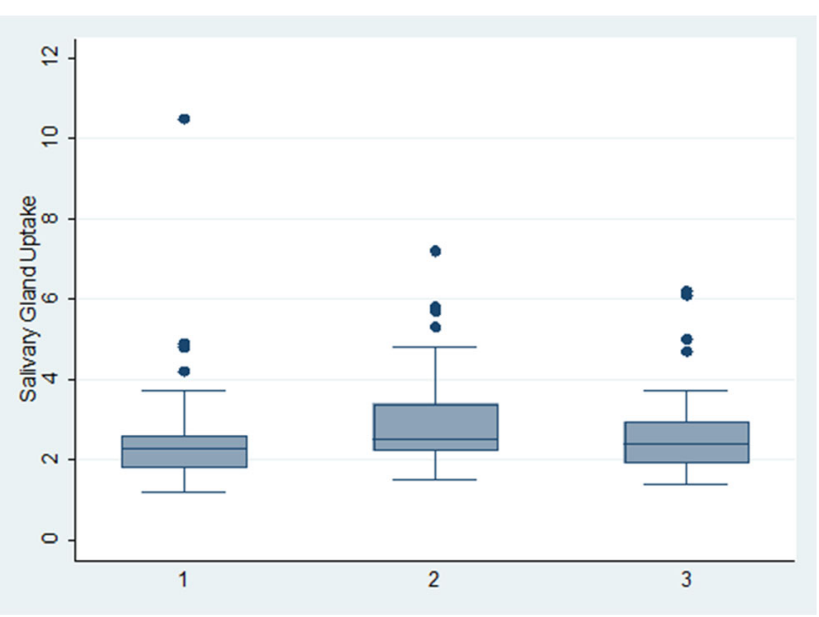

Fig. 3 Boxplot of salivary gland uptake, in patients across the 3 weeks of study time period

\section{Discussion}

To our knowledge, this is the largest single centre retrospective controlled observational study in patients undergoing 18F-FDG PET/CT scans during the first 3 weeks of COVID-19 lockdown phase in the UK. The data obtained could further inform the use of 18F-FDG PET/CT as an imaging tool to document extent of pulmonary and nonpulmonary organ involvement. There are several key findings from our study: First, the total prevalence of incidental 18F-FDG PET/CT findings was $16.25 \%$. Lung-related incidental 18F-FDG PET/CT findings consistent with suspicious for COVID-19 were found in 15/160 (9.3\%) and extrapulmonary incidental findings were found in 11/160 (7\%) patients. Second, the ascending trend in the prevalence of incidental 18F-FDG PET/CT findings was parallel to the trend of confirmed COVID-19 cases in the community during the first 3 weeks of lockdown. Third, there was no significant difference in the prevalence of incidental findings compared to the control group, but the number of pulmonary vs. extrapulmonary findings was different

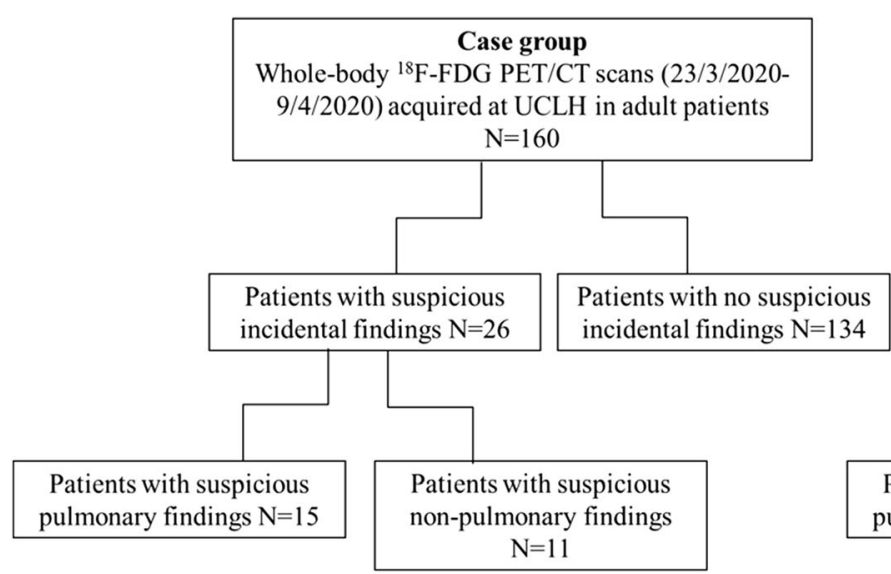

between the two populations. Fourth, there was no significant association of the appearance of incidental findings (pulmonary and non-pulmonary) with gender, age or ethnicity. Fifth, the average SUVmax of the parotid and salivary glands was significantly higher during the 2nd week in comparison with the 1 st week. No other statistically significant temporal change in the average SUVmax measured at the remaining extrapulmonary sites was observed. There was no significant difference in SUVmax values of the control group.

The COVID-19 pandemic has left its mark worldwide and the global healthcare community continue to learn lessons from this infectious disease. Our retrospective study was designed to record incidental $18 \mathrm{~F}-\mathrm{FDG}$ PET/CT findings in patients during the first 3 weeks of the COVID-19 government lockdown in the UK. The vast majority of patients were asymptomatic or mildly symptomatic with symptoms not strongly associated with COVID-19 [6] compliant with local and UK government guidance for performing these studies on an urgent basis [2].

In our study of 160 adult patients, we observed a total prevalence of incidental findings of $16.25 \%$ of which 9.3\% were lung-related compatible with COVID-19 pulmonary manifestations and $7 \%$ with other incidental findings such as PE, abnormal cardiac uptake, increased FDG uptake in the parotid glands and the tonsils and signs of increased upper gastrointestinal uptake and colitis known extrapulmonary organs affected by COVID-19 on imaging [11]. The published literature on the role of $18 \mathrm{~F}-\mathrm{FDG}$ $\mathrm{PET} / \mathrm{CT}$ value during the COVID-19 pandemic is scarce and consists mainly of sporadic case reports and a larger series of 65 asymptomatic patients from Italy and 129 from Spain, which showed incidental pulmonary findings in six $(9 \%)$ and eleven $(8.5 \%)$ of patients compatible with COVID-19 respectively $[15,16]$. Although extrathoracic symptoms have been described [4], there are no comprehensive publications for COVID-19-related extrathoracic imaging findings on $18 \mathrm{~F}-\mathrm{FDG}$ PET/CT. To the best of our

Patients with suspicious pulmonary findings $\mathrm{N}=12$

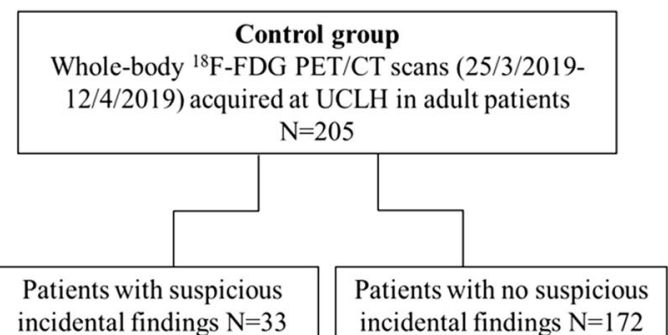

incidental findings $\mathrm{N}=33$ incidental findings $\mathrm{N}=172$

Fig. 4 Case-control comparison of frequency and pattern of suspicious 18F-FDG PET/CT scans (see results) 
knowledge, the only relevant studies describe cardiac findings and abdominal findings on CT [11, 17].

To support our hypothesis that the reported incidental findings could be attributed to the high prevalence of SARS-CoV2 spread in the community (on the rise in the designated time period), our analysis revealed an ascending trend in the prevalence of incidental findings discovery in our cohort, parallel to the trend of confirmed COVID-19 cases in the community (Fig. 2). Specifically, our analysis showed that there was a significantly higher chance of the appearance of incidental findings in the patients who were scanned in the 2nd $(\mathrm{OR}=$ $3.8)$ and the 3 rd week $(\mathrm{OR}=7.6)$ of the lockdown in comparison with patients scanned during the 1 st week. This trend of increase in incidental findings was not observed in the control group of patients in our study.

The pattern and type of 18F-FDG PET/CT incidental findings in a pre-pandemic date-matched control group from 2019 was also evaluated. The total prevalence of incidental findings in the control group $(16.1 \%)$ was similar to the total prevalence reported in the case group. However, the prevalence of

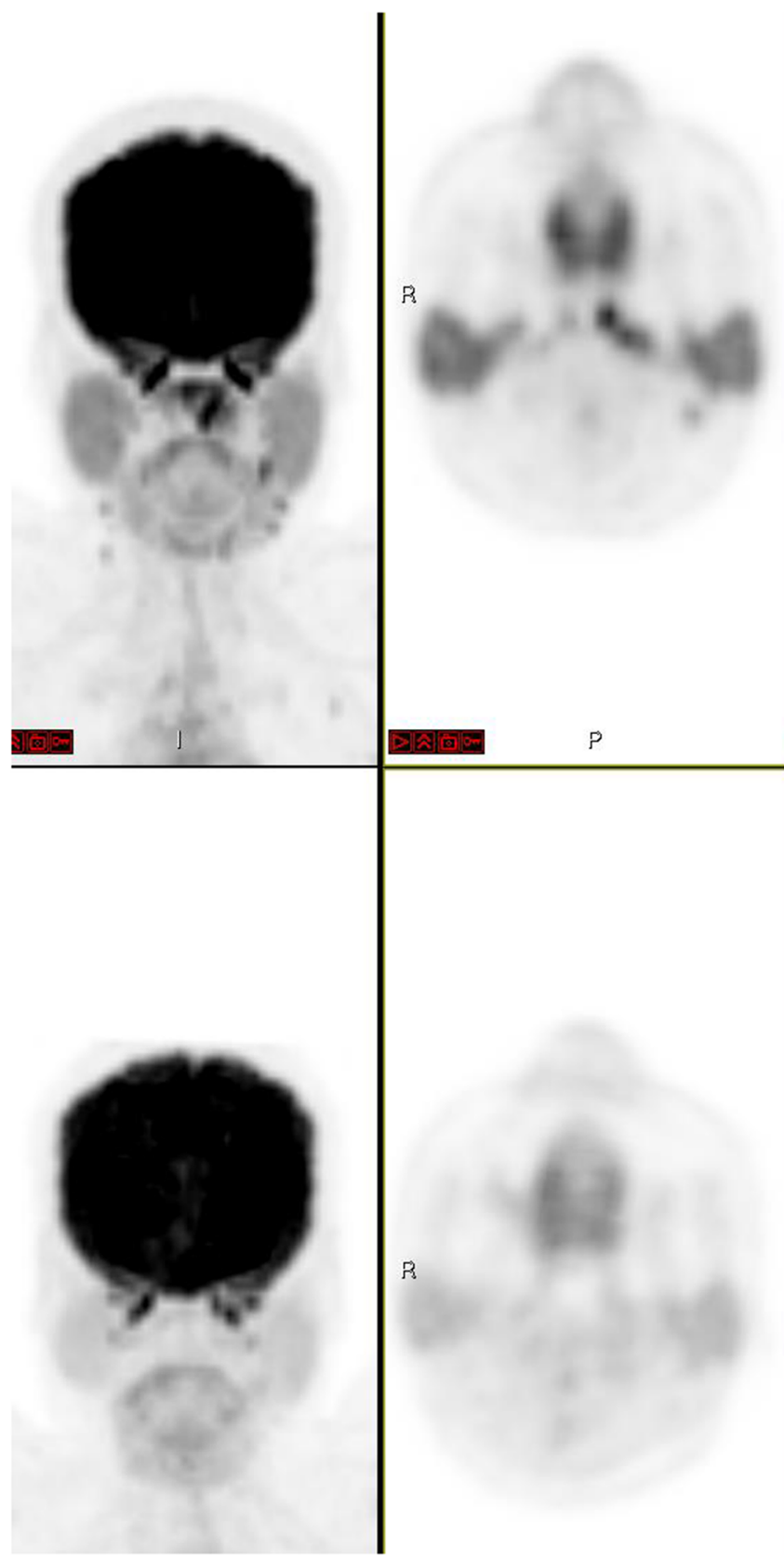

Fig. 5 Two 18F-FDG PET/CT scans of 36-year-old woman for restaging of plasmablastic lymphoma with no recent treatment. The scans were performed two months apart on the same camera. Current scan (top row) performed during the UK lockdown demonstrating increased

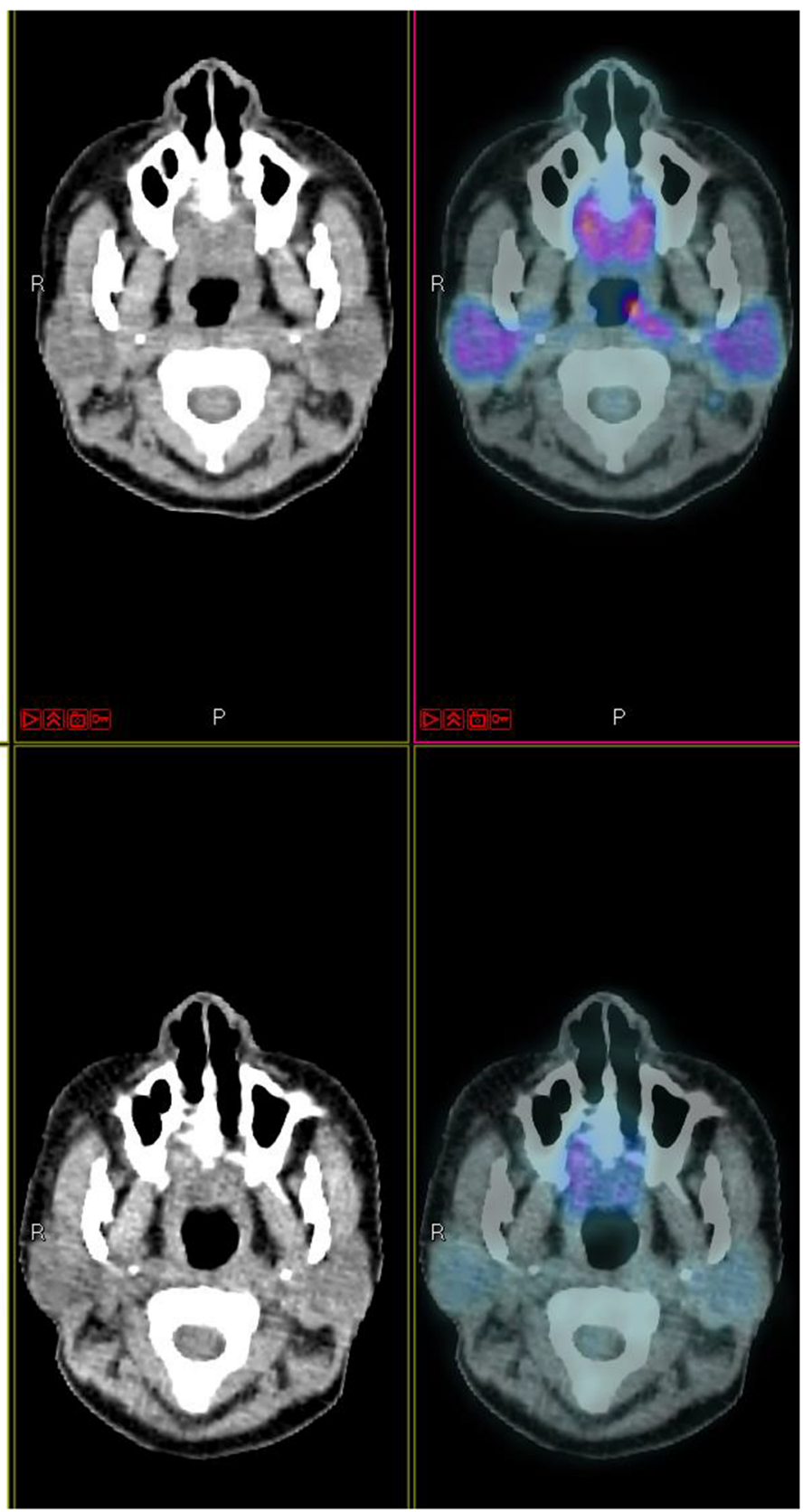

parotid and tonsillar uptake compared to the previous study (bottom row). (SUVmax 6.1 and 5.2 vs. 3.2 and 3.2 respectively). Reference values for the two studies were similar: liver and mediastinal blood pool SUVmax 3/1.8 and 3.3/1.9 respectively 


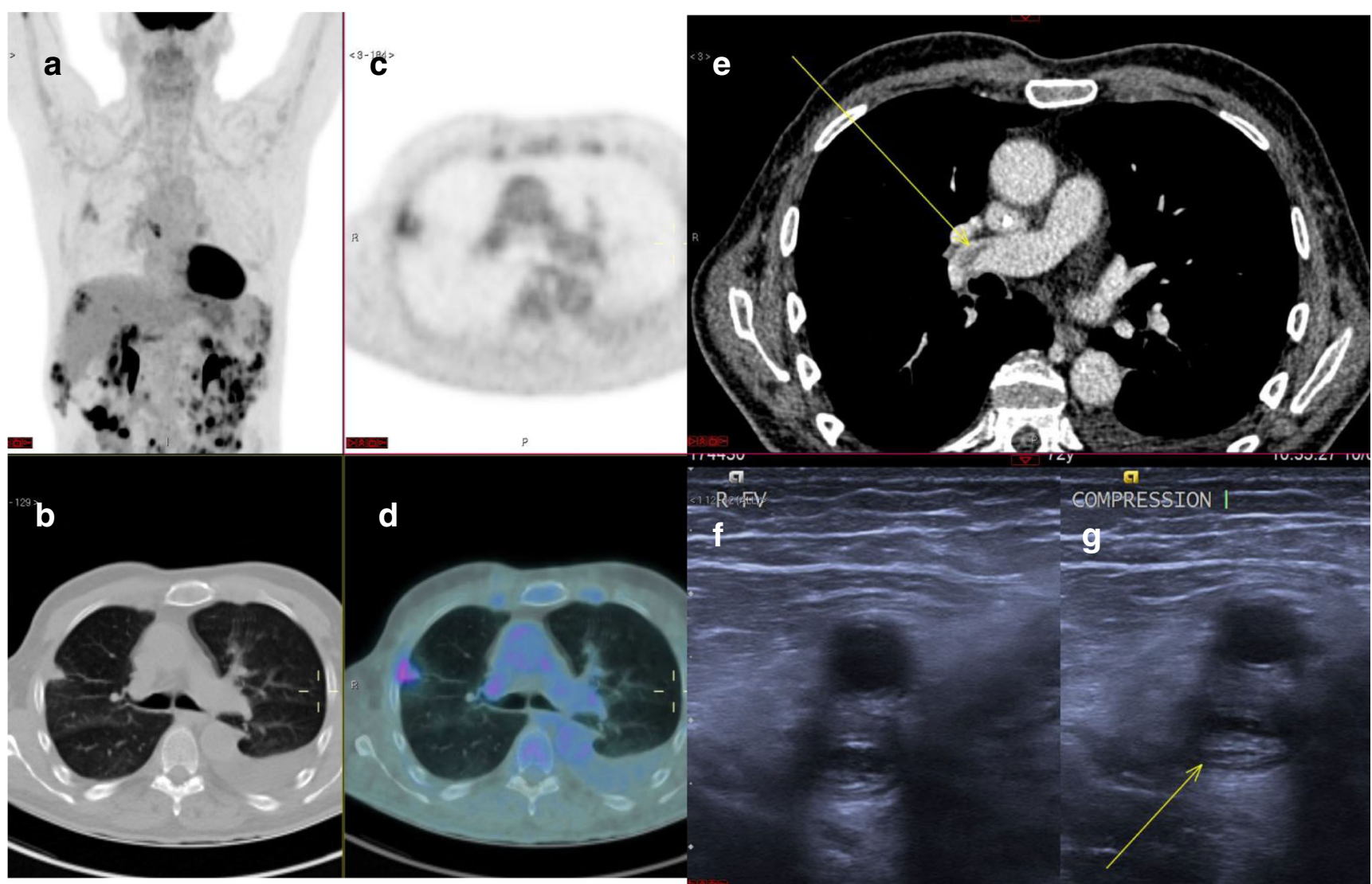

Fig. 6 Seventy-year-old man, with diffuse large B cell lymphoma due for novel immunotherapy. Panels a-d show ${ }^{18}$ F-FDG PET/CT scan, demonstrating one of two avid (SUVmax 3.1) peripheral, wedgeshaped areas of pulmonary consolidation in right lung. Panel e shows CTPA performed 2 days later confirms bilateral semi-occlusive acute

lung-related manifestations was only $5.8 \%$ in the control group. It is reasonable to speculate that the excess occurrence of incidental pulmonary findings in our test group from this year is at least in part secondary to patients with undiagnosed, subclinical COVID-19. The presence of incidental lung findings similar to COVID-19 manifestations in a subset of the control group may be attributed to a range of differential diagnoses with similar CT features (i.e. predominantly mid/ lower lobe subpleural ground glass change and consolidation), such as other viral pneumonia (e.g. seasonal flu), or druginduced pneumonitis [18].

Our study is the first attempt to explore and include incidental findings beyond the lungs as there is a growing body of evidence of non-respiratory manifestations of SARS-CoV-2 infections. Extrathoracic findings on 18F-FDG PET/CT were observed in $7 \%$ of the case group. This consisted of increased tonsillar, salivary gland and gastrointestinal uptake which are occasional and recognised variants observed with $18 \mathrm{~F}-\mathrm{FDG}$ $\mathrm{PET} / \mathrm{CT}$ and may be related to infection from SARS-CoV-2 in accordance with the virus's mechanisms of entry, the range of related symptoms and emerging evidence from published cases $[4,11,15,17,19,20]$. The correlation of extrathoracic pulmonary emboli within the distal main pulmonary arteries (right side shown). Panels f-g show peripheral lower limb Doppler ultrasound, demonstrating occlusive, non-compressive, echogenic, long segment deep vein thrombus in the right leg (arrowed)

incidental findings with symptoms and the attribution to SARS-CoV-2 infection cannot be consistently supported by our data due to the inclusion of asymptomatic patients and the low availability of PCR testing. There is, however, temporal correlation which may be better illustrated in isolated cases, such as the one presented in Fig. 5 where new increased parotid uptake can be seen in comparison with recent 18F-FDG $\mathrm{PET} / \mathrm{CT}$.

There were no patients with incidental thrombotic, cardiac or large vessel 18F-FDG PET/CT abnormalities. A recent study in 113 COVID-19 patients reported an incidence of DIC of $8 \%$ [21]. In our study, we only observed one case of thrombotic complication in the study cohort (Fig. 6). Evidence of the association of a highly activated coagulation system on survival outcomes is also emerging. A retrospective study of 171 patients admitted to a hospital in Wuhan with COVID-19 found that elevated D-dimer $(>1000 \mu \mathrm{g} / \mathrm{L})$ was an independent risk factor for mortality in multivariate analysis and was associated with an 18-fold increased risk of death [12]. Going forward, it is likely that these manifestations will be noted in patients with comorbidities, mainly cancer patients who are undergoing 18F-FDG PET/CT scans. 
Fig. 7 A 62-year-old man, 18FFDG PET/CT (coronal reformat) for pyrexia unknown origin, history of a cough and fever two days prior to the study. The scan shows diffuse small and large bowel 18F-FDG uptake; patient was not on metformin or other treatment. The patient had a negative SARS-CoV-2 PCR test 1 day prior to the study, subsequently had a positive test 2 days after the PET/CT and developed gastrointestinal symptoms (diarrhoea/loose stools for 5 days)

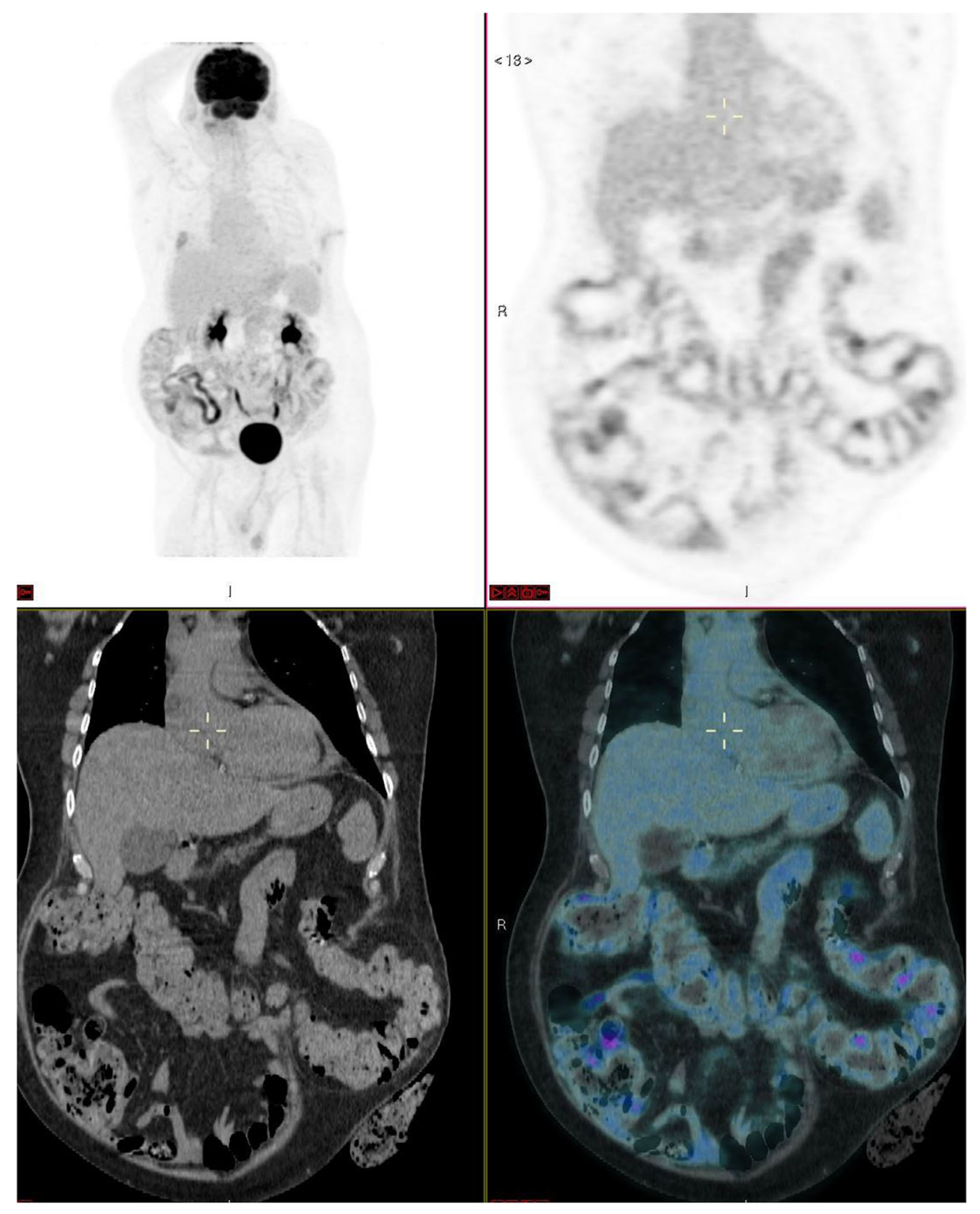

In our cohort of patients, we did not observe any significant correlation of incidental 18F-FDG PET/CT findings in asymptomatic patients and demographic risk factors (gender, age or ethnicity). The UK cohort analysis of patient deaths by the OpenSAFELY initiative has quantified several demographic risk factors for adverse outcomes in COVID-19 infection, including male sex, increasing age, black and ethnic minority ethnicity (BAME) status, as well as socioeconomic factors such as deprivation and predisposing medical conditions, such as severe asthma or uncontrolled diabetes [22].

To further study the potential correlation of FDG uptake in extrathoracic organs with the trend of the SARS-CoV-2 infection in the community, we measured SUVmax values in extrathoracic organ sites. We observed a significant increase of parotid/salivary gland FDG uptake in patients scanned during the 2 nd week compared to patients scanned during the 1 st week. This is in accordance with a published hypothesis that SARS-CoV-2 may cause acute sialadenitis due to increased ACE-2 receptor expression in the salivary glands, supported by the observation that the virus can be detected in saliva [23]. This may also be followed by symptoms of discomfort, pain or swelling of the salivary glands; however, these symptoms may be overlooked during established COVID-19 assessment thus far, which is mostly based on fever and respiratory symptoms. No significant SUVmax change with time was observed in any other extrapulmonary organs.

To our knowledge, there are no published case controlled, observational 18F-FDG PET/CT studies which coincide with 
the phase of accelerating SARS-CoV-2 transmission in the UK community. The relatively large sample of patients, systematic and structured reporting of incidental findings, investigation of available clinical data on extrapulmonary findings, semiquantitative data (SUVmax) analysis and comparison with a large date-matched control group from 2019 (preCOVID-19) further highlights the spectrum of emerging expectant findings in minimally symptomatic or asymptomatic patients during the pandemic.

Our study was observational and limitations include retrospective nature of study, absence of reliable rapid universal testing for SARS-CoV-2 viral status during the study and lack of sufficient long-term follow-up data to confirm that all our observations were correlated with SARS-CoV-2 infection. The low availability of SARS-CoV-2 PCR testing during the early stages of the pandemic (prioritised for symptomatic patients only) was a factor which was beyond our control. The lockdown measure itself introduces a bias, as patients with any perceived symptoms even mild ones may choose to stay home and postpone their scan.

\section{Conclusion}

This study provides a useful, novel base of evidence to highlight the incidental 18F-FDG PET/CT findings in asymptomatic patients and in patients with minor symptoms without clinical suspicion of COVID-19 during the pandemic. This may educate and increase awareness of expectant findings in should there be further waves of the disease [24] and inform further studies on the utility of molecular imaging in COVID19 [25]. Moreover, with the prospect of SARS-CoV-2 becoming an endemic virus, it may well be perpetually necessary to include this infection in the list of differential diagnoses when faced with relevant pulmonary or extrapulmonary incidental findings on 18F-FDG PET/CT studies.

Acknowledgements Prof Bomanji and Dr. Wan acknowledge support from the NIHR Biomedical Centre at UCL Hospitals NHS Foundation Trust and support with research governance from Robert Shortman. Dr. Halsey thanks Dr. Vivienne Eze for her assistance in early draft.

\section{Compliance with ethical standards}

Conflict of interest The authors declare that they have no conflict of interest.

Role of funding source and oversight 18F-FDG PET/CT scans were performed as standard of care. Data was accessed centrally from the hospitals PACS and Epic information systems. None of the investigators received any payments.

Ethical approval IRAS project 286154 has received ethical approval from the NHS Health.
Research authority Exception from Research Ethics Committee review applies to research undertaken by staff within a care team using information previously collected in the course of care for their own patients, provided that data is anonymised in conducting the research. All procedures performed on participants were in accordance with ethical standards of the local institution and/or national research committee and in line with the 1964 Helsinki Declaration and its later amendments.

\section{References}

1. PM address to the nation on Coronavirus: 23 March 2020 [press release]. Prime Minister's Office, 10 Downing Street 2020.

2. Annunziata S, Bauckneht $M$, Albano D, et al. Impact of the COVID-19 pandemic in nuclear medicine departments: preliminary report of the first international survey. Eur J Nucl Med Mol Imaging. 2020. https://doi.org/10.1007/s00259-020-04874-z.

3. Huang HL, Allie R, Gnanasegaran G, Bomanji J. COVID19nuclear medicine departments, be prepared! Nucl Med Commun. 2020;41:297-9.

4. Vetter P, Vu DL, L'Huillier AG, Schibler M, Kaiser L, Jacquerioz F. Clinical features of covid-19. BMJ. 2020;m1470..

5. Paez D, Gnanasegaran G, Fanti S, Bomanji J, Hacker M, Sathekge M, et al. COVID-19 pandemic: guidance for nuclear medicine departments. Eur J Nucl Med Mol Imaging. 2020;47:1615-9.

6. Public Health England Guidance: Stay at home: guidance for households with possible or confirmed coronavirus (COVID-19) infection (Updated 18 June 2020), www.gov.uk/government/ publications/covid-19-stay-at-home-guidance/stay-at-homeguidance-for-households-with-possible-coronavirus-covid-19infection.

7. Boellaard R, Delgado-Bolton R, Oyen WJG, Giammarile F, Tatsch $\mathrm{K}$, Eschner W, et al. FDG PET/CT: EANM procedure guidelines for tumour imaging: version 2.0. Eur J Nucl Med Mol Imaging. 2015;42:328-54.

8. Shielded patients list guidance for hospital trusts [NHS Digital. [cited 2020 May 26]. Available from: https://digital.nhs.uk/ coronavirus/shielded-patient-list/spl-guidance-for-hospitals

9. UPDATED BSTI COVID-19 Guidance for the Reporting Radiologist. The British Society of Thoracic Imaging. [cited 2020 May 26]. Available from: https://www.bsti.org.uk/standardsclinical-guidelines/clinical-guidelines/bsti-covid-19-guidance-forthe-reporting-radiologist/.

10. Zhu J, Zhong Z, Li H, Ji P, Pang J, Li B, et al. CT imaging features of 4121 patients with COVID-19: a meta-analysis. J Med Virol. 2020;jmv.25910.

11. Bhayana R, Som A, Li MD, Carey DE, Anderson MA, Blake MA, et al. Abdominal imaging findings in COVID-19: preliminary observations. Radiology. 2020;201908.

12. Zhou F, Yu T, Du R, Fan G, Liu Y, Liu Z, et al. Clinical course and risk factors for mortality of adult inpatients with COVID-19 in Wuhan, China: a retrospective cohort study. Lancet. 2020;395: 1054-62.

13. Wang S, Zhou X, Zhang T, Wang Z. The need for urogenital tract monitoring in COVID-19. Nat Rev Urol. 2020 [cited 2020 May 26]; Available from: http://www.nature.com/articles/s41585020-0319-7.

14. Coronavirus (COVID-19) in the UK. [cited 2020 May 26]. Available from: https://coronavirus.data.gov.uk/.

15. Albano D, Bertagna F, Bertoli M, Bosio G, Lucchini S, Motta F, et al. Incidental findings suggestive of COVID-19 in asymptomatic patients undergoing nuclear medicine procedures in a highprevalence region. J Nucl Med. 2020;61:632-6.

16. Mucientes Rasilla J, Jimeno Pernett R, Cardona Arboniés J. Diagnosis of COVID-19 pneumonia in asymptomatic patients after 
an oncological PET/CT published online ahead of print, 2020. Rev Esp Med Nucl Imagen Mol. 2020;S2253.

17. Inciardi RM, Lupi L, Zaccone G, Italia L, Raffo M, Tomasoni D, et al. Cardiac involvement in a patient with coronavirus disease 2019 (COVID-19). JAMA Cardiol. 2020 [cited 2020 May 26]; Available from: https://jamanetwork.com/journals/ jamacardiology/fullarticle/2763843.17.

18. Hani C, Trieu NH, Saab I, et al. COVID-19 pneumonia: a review of typical CT findings and differential diagnosis. Diagn Interv Imaging. 2020;101(5):263-8. https://doi.org/10.1016/j.diii.2020. 03.014.

19. Zou S, Zhu X. FDG PET/CT of COVID-19. Radiology. 2020;200770.

20. Qin C, Liu F, Yen T-C, Lan X. 18F-FDG PET/CT findings of COVID-19: a series of four highly suspected cases. Eur J Nucl Med Mol Imaging. 2020;47:1281-6.

21. Chen T, Wu D, Chen H, Yan W, Yang D, Chen G, et al. Clinical characteristics of 113 deceased patients with coronavirus disease 2019: retrospective study. BMJ. 2020;m1091.

22. The OpenSAFELY Collaborative, Williamson E, Walker AJ, Bhaskaran KJ, Bacon S, Bates C, et al. OpenSAFELY: factors associated with COVID-19-related hospital death in the linked electronic health records of 17 million adult NHS patients. Epidemiology. 2020. Available from: https://doi.org/10.1101/ 2020.05.06.20092999.

23. Wang C, Wu H, Ding X, Ji H, Jiao P, Song H, et al. Does infection of 2019 novel coronavirus cause acute and/or chronic sialadenitis? Med Hypotheses. 2020;140:109789.

24. Ali I. COVID-19: are we ready for the second wave? Disaster Med Public Health Prep. 2020;1-3.

25. Nasoodi A, Johnston C, Fraioli F. COVID-19; beyond the obvious: how do we move forward? Eur J Nucl Med Mol Imaging. 2020.

\section{Research in context}

Study question This study is a retrospective analysis of imaging data of patients who underwent 18F-FDG PET/CT at University College London Hospital (UCLH) during the first 3 weeks of the UK coronavirus lockdown. The study aims to describe the demographic, clinical and 18-F-FDG PET/CT features of patients who underwent 18F-FDG PET/CT scan in UCLH, a large teaching hospital in London designated as a COVID-19 hub. Specifically, we attempt to describe the spectrum of thoracic and extrathoracic incidental findings, their potential correlation with the rising prevalence of SARS-
CoV-2 infection in the community and the potential impact on patient management.

The principal research question is whether there was an increase of incidental findings on 18F-FDG PET/CT scans during the COVID-19 pandemic which could raise the suspicion of SARS-CoV-2 infection in patients with no symptoms at all or with no typical manifestations of the infection.

Evidence before this study As it has become known that a significant proportion of infected individuals are asymptomatic but can still spread the infection, the research intends to help radiologists and nuclear medicine physicians identify those individuals as early as possible.

There is a scarcity of published data relevant to $18 \mathrm{~F}-\mathrm{FDG}$ PET/CT features of SARS-CoV-2 infection. A few sporadic case reports and limited case series focusing on lung imaging findings are available.

Added value of this study Our study provides a structured review of evidence gathered during the rise of the pandemic to inform diagnostic decisions on a patient-per-patient level, which may influence management as well as infection control practices and policies on a departmental/hospital level.

The positive findings of the study include a temporal increase in the frequency of incidental 18F-FDG PET/CT findings in asymptomatic patients, parallel to the spread of the coronavirus in the community. Apart from pulmonary findings, lessstudied extrapulmonary incidental findings assigned to COVID-19 are described, including potentially serious manifestations, such as thromboembolic complications.

Implication of generated evidence We anticipate that the results of our study will inform on better identifying COVID19-related 18F-FDG PET/CT findings and consequently on patient management, and more effective mitigation of the virus spread and increase hospital staff safety.

Publisher's note Springer Nature remains neutral with regard to jurisdictional claims in published maps and institutional affiliations.

\section{Affiliations}

\section{Richard Halsey ${ }^{1}$ (1) - Dimitrios Priftakis ${ }^{1} \cdot$ Strachan Mackenzie ${ }^{2} \cdot$ Simon Wan $^{1} \cdot$ Laura M. Davis $^{1} \cdot$ David Lilburn $^{1}$. Andrew Thornton ${ }^{1} \cdot$ Nikolaos Papathanasiou $^{3} \cdot$ Gopinath Gnanasegaran ${ }^{4} \cdot$ Jamshed Bomanji $^{1}$}

1 Institute of Nuclear Medicine, University College London Hospital NHS Foundation Trust, 235 Euston Road, London NW1 2BU, UK

2 Department Haematology, University College London Hospital NHS Foundation Trust, 235 Euston Road, London NW1 2BU, UK
3 Nuclear Medicine and PET/CT Department, University Hospital of Patras, Rio, 26504 Patras, Greece

4 Department of Nuclear Medicine, Royal Free Hospital, Pond Street, Hampstead, London NW3 2QG, UK 